\title{
Enseñanza de los Procesos en Ingeniería Software -vs- Competitividad de Empresas Creadas por Ingenieros Informáticos
}

\author{
Marco Villalobos-Abarca(1), Vesna Karmelic-Pavlov(2), Mauricio Néspolo-Cova ${ }^{(3)}$ \\ Universidad de Tarapacá, (1) Área de Ingeniería en Computación e Informática, (2) Escuela de Psicología y \\ Filosofía, (3) Escuela Universitaria de Administración y Negocios, 18 de Septiembre 2222, Arica-Chile \\ (e-mail: mvillalo@uta.cl; vkarmeli@uta.cl; mnespolo@uta.cl)
}

Recibido Jul. 6, 2015; Aceptado Sep. 7, 2015; Versión final Oct. 23, 2015, Publicado Feb. 2016

\begin{abstract}
Resumen
Se presentan los resultados de un estudio cuyo objetivo fue describir la relación percibida entre la enseñanza de los procesos de Ingeniería de Software y el nivel de competitividad alcanzado por las empresas de desarrollo de software creadas por graduados de la carrera de Ingeniería en Computación e Informática de la Universidad de Tarapacá en Chile. Para ello, se evaluó el nivel de competitividad de las empresas participantes del estudio. También se diseñó un instrumento para explorar la relevancia atribuida por los graduados a la enseñanza de los distintos procesos de ingeniería de software para la competitividad de sus empresas. Los resultados permiten distinguir cuatro procesos incluidos en la formación de pregrado que son identificados como relevantes en la formación de empresas competitivas. Entre estos destacan cliente, ingeniería, gestión de proyecto y gestión de proceso.
\end{abstract}

Palabras clave: competitividad, empresas-software, procesos, enseñanza, ingeniería-software

\section{The Teaching of Software Engineering's Processes -vs- Competitiveness of Business Created by Informatics' Engineers}

\begin{abstract}
This paper presents the results of a study done with the objective of describing the relationship between the teaching of process of Software Engineering and the level of competitiveness reached by business created by graduated students from Computation and Informatics Engineering of the University of Tarapacá in Chile. To achieve this, the level of competitiveness of the enterprises involved in the study was evaluated. Also, an instrument was designed to evaluate how relevant was the teaching of software engineering's process for graduated students, related to the competitiveness of their business. The results allow distinguishing four relevant processes during the undergraduate academic's formation, which directly influence the creation of competitive business. Worthy to mention among these are: client, engineering, project management and process management.
\end{abstract}

Keywords: competitiveness, business-software, processes, teaching, engineering-software 


\section{INTRODUCCIÓN}

El desarrollo de software se ha convertido en una actividad especializada, que ha dado origen a compañías cuya actividad fundamental es el desarrollo de software, permitiendo que las empresas de producción o servicio se dediquen a las actividades orientadas hacia sus objetivos organizacionales (Anaya, 2005). Este fenómeno creó una nueva actividad económica alrededor de la industria del software que hoy se considera promisoria para los países latinoamericanos (Anaya, 2005; Bastos y Silveira, 2009; Wilson et al., 2010). Sin embargo, los estudios coinciden en afirmar que las empresas de software latinoamericanas están en desventaja frente a los niveles de competitividad exigidos a nivel internacional (Mayer, 2004; Soto 2014).

En Chile, en general se ha perdido competitividad, ya en el 2011 se habían perdido 5 puestos en el ranking mundial, según un estudio realizado por "The Economist Intelligence Unit for Business Software Alliance", nuestro país se ubicó en el puesto 32. Pese a esto, lidera el ranking de América Latina. Este estudio fue reportado en (EMOL. Ciencia y Tecnología, 2011). Por tanto, se puede concluir que en general que las empresas de desarrollo o mantenimiento, efectivamente, como lo señalan Mayer, 2004, están en desventaja competitiva. Este diagnóstico global también incluye, obviamente, a las empresas creadas por ex - alumnos de la carrera de Ingeniería Civil en Computación e Informática (ICCI) de la Universidad de Tarapacá, Arica (UTA), Chile.

En el contexto señalado, es que aparece como relevante el conocer los factores y variables que dan cuenta del nivel de competitividad alcanzado por las empresas creadas por graduados, lo que permitiría mejorar la enseñanza de los procesos en Ingeniería de Software y potenciar las oportunidades de constitución de empresas exitosas y competitivas en el rubro por futuros egresados. Lo anterior implicaría un mejor posicionamiento, mejores ingresos, aumento en la oferta laboral y demanda por los egresados de la institución y adicionalmente, favorecer el que los graduados visualicen como una oportunidad de desarrollo profesional la creación de empresas en su especialidad.

El término competitividad en la actualidad se aplica a una empresa, a un sector económico o a un país (Hernández, 2004; Arriagada y González, 2012; Abdel y Romo, 2005; Cabrera et al., 2011). Esta investigación se centró en el nivel de empresa, pero específicamente en empresas dedicadas al desarrollo y/o mantenimiento de software que hoy son una gran oportunidad para aportar al desarrollo del país. Uno de los desafíos fue determinar una definición funcional de la competitividad a nivel empresarial y contar con instrumentos para su medición. Se realizaron análisis exhaustivos de las definiciones e instrumentos existentes. Se seleccionaron y examinaron las definiciones y modelos de competitividad más representativos a nivel empresarial y haciendo uso de una técnica de "clasificación y numeración de referencias", se eligieron los factores y variables claves que se tomaron en cuenta para la elaboración de una definición y selección de un instrumento adecuado.

Se seleccionó la propuesta de las autoras Arriagada y González (2012), para medir los aspectos más cuantitativos de la competitividad (factores influyentes) y se diseñó un instrumento para evaluar, de acuerdo a la percepción de los graduados empresarios, la atribución de relevancia a la enseñanza de los distintos procesos de ingeniería de software para la competitividad alcanzada por su empresa. El diseño se validó mediante entrevistas preliminares a un subconjunto de graduados empresarios de la Región de AricaParinacota, ciudad de Arica, Chile.

Las encuestas fueron aplicadas a 9 empresas que incluyeron a 14 graduados empresarios. Las empresas están mayoritariamente ubicadas en Arica, lquique y Santiago. Los resultados de la investigación revelan que es posible identificar la existencia de al menos 4 procesos que se enseñan en Ingeniería de Software como influyentes en la formación de empresas competitivas por parte de graduados de la citada carrera, entre los que destacan son: "cliente", "ingeniería", "gestión de proyecto" y "gestión de proceso". El estudio empírico se realizó aplicando dos cuestionarios. Con los análisis y resultados, se diseñó un nuevo curso de Ingeniería de Software, de manera tal que fue orientado no sólo a la formación profesional, sino que a la mejora de las capacidades de los egresados para crear empresas propias más competitivas en el ámbito de la especialidad.

Existe una brecha marcada entre lo que se enseña en el aula de clase y la realidad del desarrollo de software en las organizaciones (Anaya, 2005). Esta situación ha motivado una reflexión en el mundo académico, acerca de las competencias y habilidades que deben ser desplegadas en los futuros desarrolladores de software y las estrategias pedagógicas que pueden ser utilizadas de manera que sus experiencias de aprendizaje estén altamente influenciadas por las prácticas, técnicas y modos de trabajo que exige el desarrollo de software de calidad a escala industrial (Stiller y LeBland, 2002; Liu et. al., 2002; Bracken, 2003; Maibaum, 2004; Gerald et al., 2008; Alsmadi y Abul-Huda, 2011). 
Uno de los objetivos de este trabajo fue hacer un primer acercamiento de tipo reflexivo, analizando y describiendo cómo la enseñanza de procesos en Ingeniería de Software (EPIS) influencian la competitividad de las empresas de desarrollo o mantenimiento de software, creadas por graduados de la carrera ICCI-UTA.

\section{COMPETITIVIDAD Y ENSEÑANZA DE PROCESOS EN INGENIERÍA DE SOFTWARE}

Al revisar la literatura relacionada con la competitividad de la Industria del Software se ha encontrado que la mayoría de la investigaciones apunta a determinar los factores que influyen en la competitividad (Bastos y Silveira, 2009; Wilson et al., 2010, Arriagada y González, 2012; Mosquera y Millán, 2013), pero no existen estudios que vinculen la formación de los procesos en Ingeniería de Software y cómo impacta en la productividad de empresas que forman graduados de una carrera de Ingeniería Informática. De esta revisión de literatura se desprendieron dos cuestiones principales ¿Qué es la competitividad y cómo se puede medir? y ¿Cómo se relaciona la formación en procesos de la Ingeniería de Software y la creación de empresas competitivas por parte de graduados de una carrera de Ingeniería Informática?.

\section{Competitividad}

Respecto de la Competitividad dos son las cuestiones que interesa discutir: su definición y sus determinantes. El término de competitividad más bien se maneja en tres niveles: país, región (o a nivel de industrias, ramas o subsectores) y empresa (Hernández, 2004; Arriagada y González, 2012; Abdel y Romo, 2005; Cabrera et al., 2011). Sin embargo, en esta investigación, interesó sólo el nivel de empresa; así en esta sección, se desarrollará una definición y otras características de la competitividad a este nivel. Solleiro y Castañón (2005) consideran que la competitividad es un concepto complejo y puede ser estudiado desde diferentes enfoques y disciplinas; por lo cual no es posible establecer una definición única. En la tabla 1, se presentan algunas de las definiciones de la competitividad a nivel de empresa.

Tabla 1. Algunas definiciones de competitividad a nivel de empresa.

Fuente: para revisión en detalle de las referencias, ver (http://ingsoftware.uta.cl/wp-content/uploads/2014/10/Informedefinicion-competitividad-v3.31.pdf)

\begin{tabular}{|c|c|}
\hline Autores & Descripción \\
\hline $\begin{array}{l}\text { Rubio y Aragón } \\
\text { (2006) }\end{array}$ & $\begin{array}{l}\text { Capacidad de una empresa para, rivalizando con otras, alcanzar una posición competitiva } \\
\text { favorable que permita la obtención de un desempeño superior a las empresas de la competencia. }\end{array}$ \\
\hline $\begin{array}{l}\text { Lall, Albadalejo y } \\
\text { Mesquita (2005) }\end{array}$ & $\begin{array}{l}\text { Las compañías compiten para captar mercados y recursos, miden la competitividad según su } \\
\text { participación relativa en el mercado o su rentabilidad y utilizan la estrategia de la competitividad } \\
\text { para mejorar su desempeño. }\end{array}$ \\
\hline $\begin{array}{l}\text { Solleiro y } \\
\text { Castañón (2005) }\end{array}$ & $\begin{array}{l}\text { Capacidad de una organización para mantener o incrementar su participación en el mercado } \\
\text { basada en nuevas estrategias empresariales, en un sostenido crecimiento de la productividad, en } \\
\text { la capacidad interempresarial para participar en negociaciones con diferentes instituciones y otras } \\
\text { compañías dentro de su ambiente, en un ambiente competitivo determinado por el sector y el } \\
\text { mercado de los consumidores y en políticas introducidas por los gobiernos nacionales y alianzas } \\
\text { económicas regionales. }\end{array}$ \\
\hline $\begin{array}{l}\text { Abdel y Romo } \\
(2005)\end{array}$ & $\begin{array}{l}\text { La empresa se ubica en el centro de todo el debate que rodea a la competitividad. La industria, la } \\
\text { región y el país pueden proporcionar un entorno que favorezca el desarrollo de las ventajas } \\
\text { competitivas, pero es la empresa, en su papel de agente económico básico, la que responderá } \\
\text { finalmente a ese entorno. Como consecuencia (...) las políticas dirigidas al mejoramiento de la } \\
\text { competitividad deben tener a la empresa como su objetivo fundamental. }\end{array}$ \\
\hline $\begin{array}{l}\text { Perdomo y Prieto } \\
\text { (2009) }\end{array}$ & $\begin{array}{l}\text { Una forma de impulsar la competitividad en la empresa es a través de los líderes, los cuales, } \\
\text { tienen la tarea de motivar a los trabajadores para que produzcan mejor, lleguen a alcanzar } \\
\text { aquellos logros o metas propuestas y cumplan los objetivos de la empresa. }\end{array}$ \\
\hline Padilla (2006) & $\begin{array}{l}\text { El concepto está vinculado directamente con la habilidad de las firmas para operar rentablemente } \\
\text { en un mercado determinado. }\end{array}$ \\
\hline Alice (1987) & $\begin{array}{l}\text { Capacidad de las empresas para diseñar, desarrollar, producir y colocar sus productos en el } \\
\text { mercado internacional en medio de la competencia con empresas de otros países. }\end{array}$ \\
\hline $\begin{array}{l}\text { Bermeo y Bermeo } \\
\text { (2005) }\end{array}$ & $\begin{array}{l}\text { El performance valorado por la capacidad que tiene para generar más valor agregado que sus } \\
\text { competidores. }\end{array}$ \\
\hline Michalet (1981) & Permanencia de las empresas en el mercado libre. \\
\hline
\end{tabular}

En cuanto al concepto de competitividad empresarial se puede plantear que éste presenta un sinnúmero de definiciones y no es fácil encontrar una en la que todos estén de acuerdo. Sin embargo, se puede concluir a 
partir de la revisión de diferentes acercamientos que los elementos comunes son, además de la participación en el mercado, la productividad, la alta calidad de los productos ofrecidos y los bajos costos. Está presente también el criterio de la innovación, liderazgo y creatividad. La determinación de los factores que inciden en la competitividad de una empresa constituye un aspecto fundamental para su posterior proceso de medición. Sin embargo, al igual que con las definiciones, existen diferentes perspectivas frente a los factores determinantes de la competitividad de la empresa. En la tabla 2, se muestran 39 determinantes que señalan 12 autores como los influyentes.

Los determinantes de la tabla 2, se obtuvieron de las diversas propuestas de 12 autores. Durante la investigación, se clasificaron y numeraron las referencias a cada determinante que hacía cada autor. Si sólo se consideran los con una frecuencia de citación igual o mayor a 3 veces, quedan sólo 14, siendo obviamente los más utilizados en los análisis de la competitividad, como se muestra en la tabla 3 . Es importante mencionar que además de las frecuencias mayor o igual a 3, que muestra esta tabla, desde el determinante "Indicadores Externos" (tabla 2), las frecuencias fueron 9 con 2 y 16 con 1 referencia.

Tabla 2. Determinantes influyentes en la competitividad empresarial

\begin{tabular}{|l|l|l|}
\hline \multicolumn{2}{|c|}{ Determinantes } \\
\hline Recursos Humanos & $\begin{array}{l}\text { Interacción Clientes con Proveedores } \\
\text { y Usuarios }\end{array}$ & Servicio \\
\hline Investigación y Desarrollo & Indicadores Externos & Internacionalización de la Empresa \\
\hline Tecnología & Aspectos Internos & $\begin{array}{l}\text { Administración de Inventarios de Materia } \\
\text { Prima y Componentes }\end{array}$ \\
\hline Mercadotecnia & Capacidades Directivas & $\begin{array}{l}\text { Integración Mercado, I+D, Diseño e } \\
\text { Ingeniería }\end{array}$ \\
\hline Calidad & Cultura & Buenas Prácticas en la Producción \\
\hline Producción & Estrategias Empresariales & Capacidad de Inversión \\
\hline Innovación & Costos & Conocimiento de las Utilidades \\
\hline Recursos Financieros & Liderazgo Interno & Productividad \\
\hline Asociatividad con otras empresas & Mercado & Fuentes de Energía \\
\hline Precios de Venta & $\begin{array}{l}\text { Condiciones Económica e } \\
\text { Institucionales del entorno productivo }\end{array}$ & Diseño, Ingeniería, y Fabricación Industrial \\
\hline Logística & Aspectos Sistémicos & Diferenciación de Productos \\
\hline Organización Interna & Aspectos Estructurales & Políticas Industriales del Sector \\
\hline Compras & Diferenciación & Procesos Internos y de la Industria \\
\hline
\end{tabular}

Tabla 3. Frecuencia del número de referencias

\begin{tabular}{|l|c|}
\hline \multicolumn{1}{|c|}{ Determinantes } & No referencias \\
\hline Recursos Humanos & 9 \\
\hline Investigación y Desarrollo & 8 \\
\hline Tecnología & 7 \\
\hline Mercadotecnia & 6 \\
\hline Calidad & 6 \\
\hline Producción & 6 \\
\hline Innovación & 5 \\
\hline Recursos Financieros & 5 \\
\hline Asociatividad con otras empresas & 5 \\
\hline Precios de Venta & 4 \\
\hline Logística & 3 \\
\hline Organización Interna & 3 \\
\hline Compras & 3 \\
\hline Interacción Clientes con Proveedores y Usuarios & 3 \\
\hline
\end{tabular}


Tabla 4. Categorías de proceso y subprocesos que se derivaron del análisis de los estándares

\begin{tabular}{|c|c|}
\hline Categoría de proceso & Subproceso \\
\hline \multirow[t]{6}{*}{ Ingeniería } & Análisis de requerimientos del sistema \\
\hline & Análisis de requerimientos del Software \\
\hline & Diseño del software \\
\hline & Construcción del software \\
\hline & Verificación y Validación del Sistema y Software \\
\hline & Mantenimiento del software y del sistema \\
\hline \multirow[t]{7}{*}{ Cliente } & Adquirir productos software y/o servicios \\
\hline & Establecer contratos \\
\hline & Identificar las necesidades del cliente \\
\hline & Realizar auditorías y revisiones conjuntas \\
\hline & Entregar e instalar el software \\
\hline & Proporcionar servicio al cliente \\
\hline & Valorar la satisfacción del cliente \\
\hline \multirow[t]{4}{*}{ Proveedor } & Selección y Control de Proveedores \\
\hline & Gestión Integrada de Proveedores \\
\hline & Gestión Cuantitativa de Proveedores \\
\hline & Gestión de Acuerdo con los Proveedores \\
\hline \multirow[t]{5}{*}{ Gestión de proyecto } & $\begin{array}{l}\text { Inicio (Necesidad de negocio que el proyecto pretende abordar, Descripción preliminar del } \\
\text { producto, Definición del gerente de proyecto identificado/asignado, Restricciones (por } \\
\text { ejemplo presupuesto, plazo, personal, etc.) }\end{array}$ \\
\hline & $\begin{array}{l}\text { Planificación (Definir el Alcance, Desarrollar Calendario, Estimar Costos, Planificar } \\
\text { Calidad, Planificar Organización, Reclutar personal, Planificar Comunicaciones, Planificar } \\
\text { Riesgos, Planificar Adquisiciones) }\end{array}$ \\
\hline & $\begin{array}{l}\text { Ejecución (Ejecución de la Ingeniería, Ejecución de la Planificación, Herramientas para } \\
\text { Desarrollar Software) }\end{array}$ \\
\hline & Control (Cambio de alcance, calendario, costos, calidad, riesgos, cambios) \\
\hline & Terminación (Cierre administrativo, Cierre del contrato) \\
\hline \multirow[t]{9}{*}{ Gestión de proceso } & Alineamiento de la organización \\
\hline & Establecimiento del proceso \\
\hline & Evaluación del proceso \\
\hline & Mejora del proceso \\
\hline & Gestión de recursos humanos \\
\hline & Infraestructura \\
\hline & Reutilización \\
\hline & Gestión de cambio tecnológico \\
\hline & Innovación y desarrollo de la organización \\
\hline \multirow[t]{7}{*}{ Soporte } & Documentación \\
\hline & Gestión de la configuración del software \\
\hline & Garantía de calidad \\
\hline & Resolución de problemas \\
\hline & Aseguramiento de la Calidad del Producto y Proceso \\
\hline & Medición y Análisis \\
\hline & Análisis de Decisiones \\
\hline
\end{tabular}

\section{Enseñanza de los Procesos en Ingeniería de Software}

El desarrollo de software implica mucho más que escribir instrucciones de programación y ejecutarlas en un computador. Se requiere cumplir los requisitos del cliente a un costo y de acuerdo a una planificación preestablecida. Para tener éxito y obtener productos de calidad, los ingenieros de software deben regirse por un proceso de desarrollo de calidad (Sommerville, 2005; Pressman, 2006; Salinas et al., 2011).

\section{El proceso de software}

El proceso, el producto, el método, las personas y la tecnología son los elementos básicos involucrados en el desarrollo de software (Pressman, 2006). Anaya (2005), considera que una adecuada "formación en la enseñanza de la ingeniería de software debe considerar de manera natural las interrelaciones que se suceden entre dichos elementos" y que de acuerdo a Pressman (2006), pp. 664, citando a Paulish y Carleton (1994), representan los "factores controlables en la mejora de la calidad del software y el desempeño organizacional". 
En la actualidad existen varios estándares internacionales que describen los procesos asociados con la producción de software e incluso existe un marco de referencia para la Ingeniería de Software que incluye los requerimientos de la práctica de desarrollo de software en la industria, el SWEBOK (Guide to the Software Engineering Body of Knowledge) (SWEBOK, 2004). Desde la versión 2004, se establecieron 11 áreas de conocimientos KA (Knowledge Area) que integran las perspectivas teóricas y prácticas de la ingeniería de software. Los estándares analizados fueron La familia ISO 9000, IEEE Standard for Developing Software Life Cycle Processes (IEEE 1074-2006), Information technology - Software life cycle processes (ISO/IEC 12207:1995), SPICE-ISO/IEC TR 15504 y CMMi (Paulk, M., et al., 1995).

En este trabajo, se identificaron las categorías de procesos y sus respectivos subprocesos que proponían estos estándares y a partir de este análisis se propuso un instrumento que permitió vincular la formación en procesos de desarrollo de software y la competitividad de las empresas creadas por graduados. En la tabla 4 se muestran las categorías de procesos y subprocesos que se derivaron del análisis de los estándares señalados.

\section{METODOLOGÍA}

El trabajo de investigación tuvo como filosofía de investigación el positivismo (el conocimiento es concreto y los fenómenos son factibles de medición), como enfoque de investigación el deductivo a través de confrontación teórica y empírica, el estudio fue de naturaleza transversal y el método de recolección de datos primarios fue a través de la aplicación de dos cuestionarios estructurados aplicados a empresarios graduados de la Universidad de Tarapacá en el sector de la Industria del Software en los meses de julio y agosto de 2014. Fundamentalmente fue una investigación exploratoria.

\section{Diseño conceptual}

Se utilizó una muestra intencionada por disponibilidad. Se contactó desde registros institucionales de graduados a aquellos que cumplían con los criterios requeridos: ser graduados de la carrera de ICCI y ser dueños o socios creadores de una empresa de desarrollo de software. A través de revisión bibliográfica se identificaron 14 determinantes de la competitividad, los que se muestran en la tabla 3. Dado que el trabajo de Arriagada y González (2012) consideró el "Diseño y Validación de Instrumento para medir el nivel de competitividad en pequeñas empresas del sector TIC de la Región Metropolitana" (Chile), incluyendo 9 de los 14 determinantes, se contactó a las autoras y gentilmente autorizaron el uso de su instrumento y técnica de análisis.

Además, se diseñó otro instrumento (con base a la tabla 4) que permitiera explorar la percepción de los graduados empresarios respecto de la relevancia atribuida a la enseñanza de los procesos de Ingeniería de Software para el nivel de competitividad alcanzado por sus empresas. En este instrumento se incluyeron también 5 determinantes no considerados en el instrumento de Arriagada y González (Tecnología, Recursos Financieros, Asociatividad con otras empresas, Organización Interna y Compras). Para cada categoría y sus respectivos subprocesos, se formularon los ítemes tal como se muestra en la tabla 5. Con los 14 determinantes, el instrumento de Arriagada y González y el instrumento adicional, se diseñó el modelo de investigación que se muestra en la figura 1.

Tabla 5. Relación de la enseñanza de los procesos de Ingeniería de Software con el nivel de competitividad de las empresas de los graduados

\begin{tabular}{|c|c|c|c|c|c|c|c|}
\hline \multicolumn{2}{|c|}{$\begin{array}{c}\text { Indique qué } \\
\text { subproceso le } \\
\text { fue enseñado }\end{array}$} & \multicolumn{2}{|c|}{$\begin{array}{c}\text { Indique cuán competente se sentía al momento } \\
\text { egresar }\end{array}$} & \multicolumn{2}{|c|}{$\begin{array}{c}\text { Indique la importancia de la enseñanza del } \\
\text { subproceso para el desarrollo competitivo de su } \\
\text { empresa }\end{array}$} \\
\hline $\mathrm{Si}$ & No & No competente & $\begin{array}{c}\text { Medianamente } \\
\text { competente }\end{array}$ & Competente & No relevante & $\begin{array}{c}\text { Medianamente } \\
\text { relevante }\end{array}$ & $\begin{array}{c}\text { Muy } \\
\text { relevante }\end{array}$ \\
\hline
\end{tabular}

\section{Técnica de análisis de datos}

Como se puede observar en la figura 1, la técnica de análisis de datos fue mediante el procesamiento de los instrumentos, obteniendo como estadísticos promedios, frecuencias, entre otros.

\section{Determinación y descripción de la muestra}

En la tabla 6, se muestran algunos datos sobre la muestra, manteniendo la confidencialidad sobre las empresas y empresarios graduados involucrados en el estudio. 


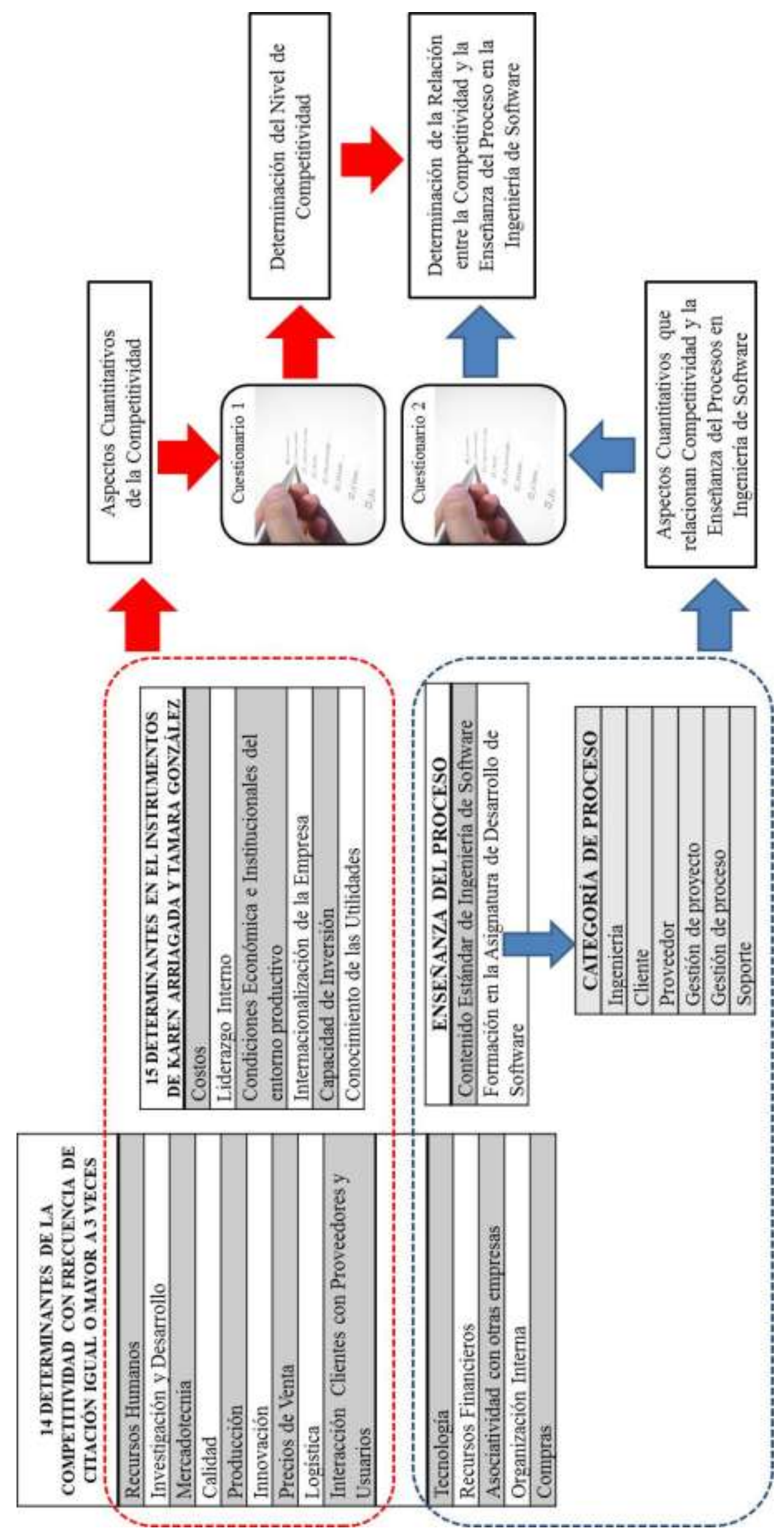

Fig. 1. Modelo de Investigación 
Tabla 6. Empresas encuestadas creadas por graduados ICCI-UTA

\begin{tabular}{|l|l|l|}
\hline Ubicación & Número de empresas & Empresarios graduados \\
\hline Arica & 3 & 4 \\
\hline Iquique & 2 & 5 \\
\hline Santiago & 4 & 5 \\
\hline Totales & 9 & 14 \\
\hline
\end{tabular}

\section{RESULTADOS}

Dos son los ámbitos de los resultados: El nivel de competitividad y la importancia que los egresados asignan a la enseñanza de los procesos (subprocesos) en el nivel competitivo de sus empresas. Respecto de lo primero, los datos indican que mientras más joven la empresa, mayor el nivel de competitividad y que 7 de 9 (78\%) son competitivas, según el instrumento de Arriagada y González (2012). De lo anterior, las relaciones entre la enseñanza de los procesos y el nivel competitivo que hacen los egresados empresarios cobran validez Así, con respecto de la relación entre enseñanza de los procesos y el nivel competitivo, varios son los resultados que pueden mencionarse:

i) De las 7 empresas competitivas, 5 (71\%) observan que todos los procesos (6) son o "Medianamente relevante" o "Muy relevante". Excepto 2 de ellas sólo indican que 1 proceso el de "Proveedores" es "No relevante" y solo 1 indica que el proceso "Gestión de Proceso" es "No relevante"

ii) La empresa menos competitiva observa que todos los procesos (6) son "Muy relevante"

iii) De las 7 empresas que son las competitivas (78\%), se observa la influencia de la enseñanza de cada proceso en el nivel de competitividad como se indica en la tabla 7.

Tabla 7. Valoración de la relación enseñanza de los procesos y la competitividad

\begin{tabular}{|c|c|}
\hline Ingeniería & $\begin{array}{l}5 \text { de } 7 \text { dice que es "Muy relevante" }(71,4 \%) \text { y } 2 \text { dicen que es "Medianamente relevante" } \\
(28,3 \%)\end{array}$ \\
\hline Cliente & 7 de 7 dice que es "Medianamente relevante" $(100,0 \%)$ \\
\hline Proveedor & $\begin{array}{l}7 \text { de } 7 \text { dice que no es "Muy relevante" }(100,0 \%), 5 \text { de } 7 \text { dice que es "Medianamente relevante" } \\
(71,4 \%) \text { y } 2 \text { dicen que es "No relevante" }\end{array}$ \\
\hline Gestión de proyecto & 5 de 7 dice que es "Muy relevante" $(71,4 \%)$ y 2 dicen que es "Medianamente relevante" $(28,3 \%)$ \\
\hline Gestión de proceso & $\begin{array}{l}6 \text { de } 7 \text { dice que no es "Muy relevante" ( } 85,7 \%), 2 \text { dicen que es "No relevante". } 4 \text { observan que } \\
\text { es "Medianamente relevante" y es "Muy relevante" a la vez }\end{array}$ \\
\hline Soporte & $\begin{array}{l}6 \text { de } 7 \text { dice que es "Medianamente relevante" }(85,7 \%), 1 \text { dice que es "No relevante". } 4 \text { observan } \\
\text { que es "Medianamente relevante" y es "Muy relevante" a la vez }\end{array}$ \\
\hline
\end{tabular}

\section{DISCUSIÓN}

En general, las empresas consideradas competitivas según el instrumento de Arriagada y González (2012), dan una alta influencia a la enseñanza de los procesos/subprocesos. Todos los procesos son influyentes excepto el proceso "Proveedor" que presenta una baja valoración llegando al 100\% opinar que "no es muy relevante". Esto ha sido analizado y una respuesta está en el tipo de "proveedor" que poseen las empresas de software y el propio tamaño de las empresas de la muestra (el $100 \%$ es una micro pyme), es decir sus ventas anuales son menores a 58 millones de pesos chilenos y tienen entre 1 y 9 trabajadores. Destacable es que todas las empresas competitivas consideran que los procesos asociados con el "cliente" e "ingeniería" son los más importantes ya que para ellas ya sea son "muy relevante" o "medianamente relevante". Esto se muestra en la tabla 8 con los promedios generales. Esto es coherente ya que la calidad del producto de software se centra fundamentalmente en la satisfacción del cliente.

Tabla 8. Promedios generales para los procesos de "ingeniería" y "cliente" según las empresas competitivas

\begin{tabular}{|l|l|l|l|}
\hline \multicolumn{4}{|l|}{ Promedio general (siete competitivas) } \\
\hline Procesos & No relevante & Medianamente relevante & Muy relevante \\
\hline Ingeniería & 0,0 & 14,3 & 85,7 \\
\hline Cliente & 0,0 & 59,2 & 40,8 \\
\hline
\end{tabular}


Por otra parte, las empresas consideradas no competitivas (2), siguen la misma valoración respecto de las competitivas. Es decir, ambas consideran en cierto grado que todos procesos son "muy relevantes", pero también, consideran que los procesos "proveedor", "gestión de proceso" y "soporte" como "no relevantes" en cierto grado. Esto explicaría en su bajo nivel de competitividad, dada la importancia que se da hoy a la gestión del proceso de software. Igual que las empresas competitivas, las no competitivas, consideran que los proceso asociados al "cliente" e "ingeniería" como los más importantes. Esto se muestra en la tabla 9 con los promedios generales. Finalmente, un análisis más detallado, muestra que todos los subprocesos, van desde un $88,9 \%$ a $100 \%$ considerados como "Medianamente relevante" o "Muy relevante". Sólo los subprocesos asociados con "proveedor", poseen una valoración que van desde un 33\% a 44\% como "no relevante" y esto se muestra en la tabla 10.

Tabla 9. Promedios generales para los procesos de "ingeniería" y "cliente" según las empresas no competitivas

\begin{tabular}{|l|l|l|l|}
\hline \multicolumn{4}{|c|}{ Promedio general (dos no competitivas) } \\
\hline Procesos & No relevante (1) & Medianamente relevante (2) & Muy relevante (3) \\
\hline Ingeniería & 0,0 & 0,0 & 100,0 \\
\hline Cliente & 0,0 & 14,3 & 85,7 \\
\hline
\end{tabular}

Tabla 10. Valoración de los subprocesos de "Proveedor"

\begin{tabular}{|l|l|l|}
\hline Proceso & Subprocesos & No relevante \\
\hline Proveedor & Selección y Control de Proveedores & $33,3 \%$ \\
\cline { 2 - 3 } & Gestión Integrada de Proveedores & $44,4 \%$ \\
\cline { 2 - 3 } & Gestión Cuantitativa de Proveedores & $44,4 \%$ \\
\cline { 2 - 3 } & Gestión de Acuerdo con los Proveedores & $33,3 \%$ \\
\hline
\end{tabular}

\section{CONCLUSIONES}

De los resultados mostrados, de su análisis y de su discusión, se pueden obtener las siguientes conclusiones: sobre la relación de la enseñanza de los procesos de la ingeniería de software y el nivel de competitividad de las empresas creadas por graduados de la carrera ICCI-UTA: 1) en general las empresas consideradas competitivas señalan que todos los procesos son influyentes, excepto el asociado con el "proveedor"; 2) Todas las empresas, tanto las competitivas como las no competitivas, consideran que los procesos asociados con el "cliente" e "ingeniería" son los más importantes; 3 ) a la luz de los resultados, se hizo necesario modificar el curso de "Ingeniería de Software" tradicional, dando énfasis a los procesos de "cliente", "ingeniería", "gestión de proyecto" y "gestión de proceso", según los estándares presentados en la sección "Enseñanza de los Procesos en Ingeniería de Software" del presente artículo.

\section{AGRADECIMIENTOS}

Este trabajo cuenta con el financiamiento del programa de Investigación en Educación de la Universidad de Tarapacá, Arica, Chile, 2013-2014, en el contexto de proyecto "Una investigación exploratoria-descriptiva de la competitividad de empresas creadas por graduados de la Carrera de Ingeniería en Computación e Informática de la UTA" (código 8720-13). Así mismo, a los graduados empresarios por su valiosa colaboración, interés y disposición. A todos y cada uno de ellos muchas gracias porque sin su colaboración esta investigación no podría haber concluido.

\section{REFERENCIAS}

Abdel, G., Romo, D., Sobre el Concepto de Competitividad. Comercio Exterior, 55(3), 200-214, (2005)

Alice, J., Evaluating industrial competitiveness at the office of technology in society. First Edition, Basic Book Inc., New York, USA, (1987)

Alsmadi, I., Abul-Huda, B., Improving Understandability In Teaching of Software Engineering and Connectivity with the Industry", IEEE EDUCON Education Engineerig 2011: Learning Environment and Ecosystems in Engineering Education Session T1A, Sumaya University, 20-25, Amman, Jordan, April, 0406, (2011) 
Anaya, R., Una visión de la enseñanza de la ingeniería de software como apoyo al mejoramiento de las empresas de software, Revista Universidad EAFIT, 42(141), 60-76, (2006)

Arriagada, K, González, T., Diseño y Validación de Instrumento para medir el nivel de competitividad en pequeñas empresas del sector TIC de la Región Metropolitana, Revista Gestión de las Personas y Tecnología, № 13, 179-189, (2012)

Bastos, P., Silveira, F., editores, Desafíos y Oportunidades de la industria del software en América Latina. 1a Edición, CEPAL en coedición con Mayol Ediciones S.A., Colombia (2009)

Bermeo, J.; Bermeo, E., Las directrices del costo como fuentes de ventajas competitivas. Estudios Gerenciales, Universidad ICESI, Cali, Colombia, 94, 81-103, (2005)

Cabrera, A., López, C., Ramírez, C., La Competitividad Empresarial: Un marco conceptual para su estudio, Documentos de Investigación, Administración de Empresas, Universidad Central, Bogotá, Colombia, № 4, (2011)

EMOL. Ciencia y Tecnología. Chile baja cinco puestos en ranking mundial de competitividad tecnológica, (2011), http://www.emol.com/noticias/tecnologia/2011/09/27/505244/chile-baja-5-puestos-en-rankingmundial-de-competitividad-tecnologica-y-se-ubica-en-el-lugar-32.html. Acceso: enero (2012)

Hernández, R., "Marco teórico-conceptual de la competitividad, (en la web: http://www.eclac.cl/mexico/noticias/paginas/5/15115/Competitividad_pres-1.pdf, 15 de mayo de 2014), CEPAL, México, (2004)

ISO/IEC 12207 Information Technology / Software Life Cycle Processes, http://www.iso.org/iso/home.html, acceso 20 mayo de 2014.

Lall, S., Albaladejo, M., Mesquita, M. La Competitividad Industrial de América Latina y el Desafío de la Globalización. Primera Edición, 1-107, BID-INTAL, Buenos Aires, Argentina (2005)

Mark C. Paulk, Charles V. Weber, Bill Curtis, and Mary Beth Chrissis. The Capability Maturity Model: Guidelines for Improving the Software Process. Edición, Reading, Addison Wesley Publishing Company, Boston, USA, (1995)

Mayer, R., Panorama de la Industria de Software en Latinoamérica, Primera edición, 97-102, MBI-Mayer \& Bunge Informática LTDA., (2004)

Michalet, C.A., Competitiveness and Internationalization, Mimeo - OCDE, Paris, Francia, (1981)

Mosquera A., Millan S., Competitividad en empresas innovadoras de software y tecnologías informáticas. Cuadernos de Administración, 29(49), 37-44. (2013)

Padilla, R., Instrumentos de medición de la competitividad, (en la web: http://www.competitividad.org.do/wpcontent/uploads/2009/01/2.1Indicadoresdecompetitividad1.pdf, 15 de mayo de 2014), CEPAL - Sede Subregional México, Santo Domingo, República Dominicana (2006)

Paulish, D., Carleton, A., Case studies of software-process-improvement measurement. Computer, 27(9), 50-57, (1994)

Perdomo, Y., Prieto, R., El liderazgo como herramienta de competitividad para la Gerencia del servicio. Revista CICAG, 6(2), 30-48, (2009)

Pressman, R. Ingeniería del Software: Un enfoque práctico. Sexta Edición, 1-960, McGraw-Hill, México, D.F., México, (2006)

Rubio, A., Aragón, A., Competitividad y recursos estratégicos en la Pyme. Revista de empresa, 17, 32-47. (2006)

Salinas, E., Cerpa, N., Rojas, P., Arquitectura orientada a servicios para software de apoyo para el proceso personal de software. Ingeniare. Revista chilena de ingeniería, 19(1), 40-52. (2011) 
Solleiro, J.; Castañón, R., Competitiveness and innovation systems: the challenges for México's insertion in the global context. Technovation, 45 (25), 1059-1070, (2005)

Sommerville, I. Ingeniería de Software. Séptima Edición, 1-687, Addison Wesley-Pearson Educación, Madrid, España, (2005)

Soto, J, Revisión de la Actividad de TI en Chile, http://www.acti.cl/files/ACTI-IDC-Indicador-Actividad-TI-enChile-2013.pdf, acceso 20 de mayo 2014. (2013)

SWEBOK, Guide to the Software Engineering Body of Knowledge. SWEBOK 2004 Version. A project of the IEEE Computer Society Professional Practices Comite. (http://www.computer.org/portal/web/swebok/)

(2001. Guide to the Software Engineering Body of Knowledge - SWEBOK. Alain Abran, Pierre Bourque, Robert Dupuis, and James W. Moore (Eds.). IEEE Press, Piscataway, NJ, USA.

(http://dl.acm.org/citation.cfm?id=580192)) (2004)

The Spice User Group, SPICE-ISO/IEC TR 15504, http://www.spiceusergroup.org, acceso 30 marzo 2014.

Wilson, F., Briones, P., Troncoso, M., González, L., La Industria Chilena de Software, http://www.inf.udec.cl/ CT-ESIC/Educacion/Prospectiva-Software.pdf, acceso 20 mayo de 2014. (2010) 
